\title{
Single lymph node metastasis 10 years after radical resection of a thymoma
}

Pietro Bertoglio, MD, ${ }^{a}$ Marco Lucchi, MD, ${ }^{a}$ Vittorio Aprile, $\mathrm{MD},{ }^{\mathrm{a}}$ Greta Alì, $\mathrm{MD}, \mathrm{PhD},{ }^{\mathrm{b}}$ and Alfredo Mussi, MD, ${ }^{a}$ Pisa, Italy

From the ${ }^{\mathrm{a} T h o r a c i c}$ Surgery, and ${ }^{\mathrm{b}}$ Pathological Anatomy, Department of Surgical, Medical and Molecular Pathology and Critical Area, University of Pisa, Pisa, Italy.

Disclosures: Authors have nothing to disclose with regard to commercial support.

Received for publication Nov 28, 2016; revisions received Feb 8, 2017; accepted for publication March 5, 2017; available ahead of print April 12, 2017.

Address for reprints: Pietro Bertoglio, MD, U.O. Chirurgia Toracica, via paradisa 2, edificio 10, Ospedale Cisanello, 56124 Pisa (PI), Italy (E-mail: pietro.bertoglio@gmail.com).

J Thorac Cardiovasc Surg 2017;154:e11-3

$0022-5223 / \$ 36.00$

Copyright (C) 2017 by The American Association for Thoracic Surgery

http://dx.doi.org/10.1016/j.jtcvs.2017.03.036

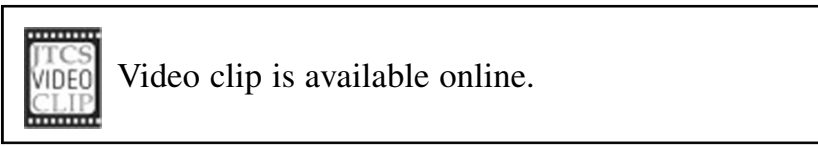

Surgery for thymoma currently is the gold standard treatment for early stages and resectable diseases. MasaokaKoga stage and R0 resection are considered the most important prognostic factors for both overall survival and disease-free interval. According to the International Thymic Malignancy Interest Group's definition, recurr-

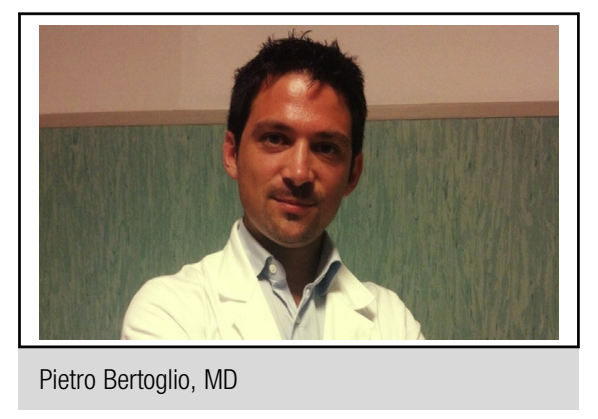

Central Message

Thymoma can recur years after the first surgical treatment. Lymph nodes might be the recurrence site in rare cases. Multimodality treatment including surgery can be offered with good results.

See Editorial Commentary page e15.

ence for thymoma can be defined as local, regional, or distant, and thymomas are reported in $4 \%$ to $36 \%$ of cases. ${ }^{1}$ We report a case of a single mediastinal lymph
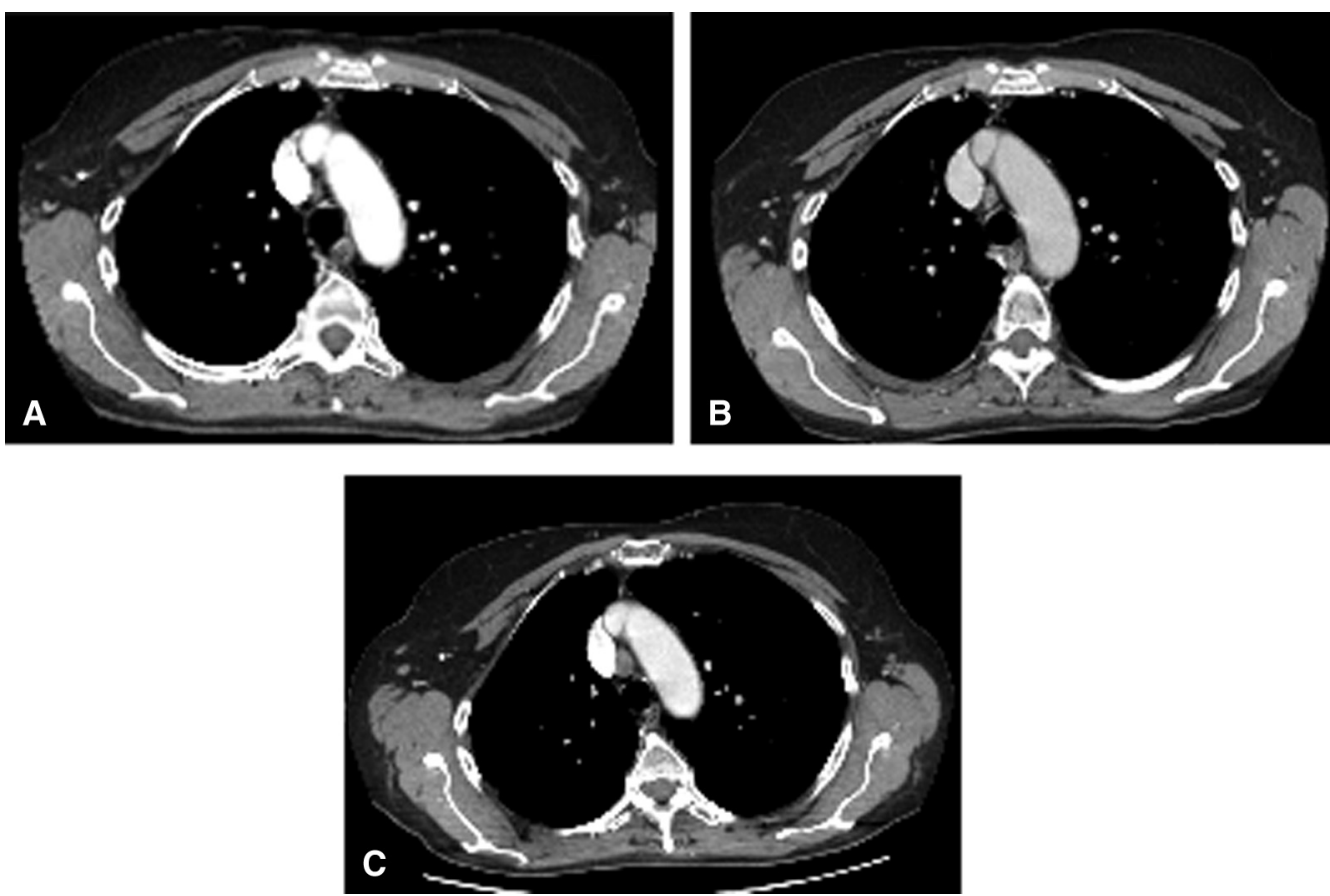

FIGURE 1. Follow-up CT scan images. A, August 2013. B, September 2014. C, December 2015. 
node metastasis 10 years after a radical resection of an invasive thymoma.

\section{CASE REPORT}

In June 2005, a 57-year-old female patient underwent chest $\mathrm{x}$-ray for pneumonia, which revealed a large mediastinal mass. The patient underwent a computed tomography (CT) scan that confirmed a 9-cm large mass in the anterior part of the mediastinum with a strict contact with the right pleural space. No mediastinal lymph node enlargement was noticed. After multidisciplinary discussion, despite the absence of myasthenic symptoms and negative titer of acetylcholine receptor antibody, a thymoma was suspected and surgical resection was planned. An extended thymectomy was performed via a right posterolateral thoracotomy because a large part of the tumor exceeded the right side. The right mediastinal pleura, but not the pericardium or mediastinal vessels, was infiltrated, and a radical resection was easily achieved. Concurrently, a lymph node sampling of the paratracheal region was performed. The patient's postoperative course was uneventful, and she was discharged on postoperative day 5 .

The patient's pathology report revealed a World Health Organization B1 thymoma with Masaoka-Koga stage III.
All of the retrieved lymph nodes showed no sign of metastasis. After a multidisciplinary discussion, the patient was sent for adjuvant radiotherapy on the mediastinum (45 Gy) on the tumor bed. The follow-up was then carried out with chest and abdomen CT scan twice per year for the first 2 years after surgery and once per year afterward (Figure 1). The patient did not experience a relapse until December 2015 (10.5 years after the first intervention), when a CT scan showed an enlarged pretracheal lymph node (Figure 1,C) that was confirmed by a positron emission tomography scan (5.9 standardized uptake value). A lymphoma due to the previous mediastinal radiotherapy was suspected, and the patient was referred again to our unit for a mediastinoscopy. Almost all of the enlarged lymph node was removed together with the surrounding tissue, and the pathologic report confirmed the diagnosis of thymoma metastasis (Figure 2). All of the available therapeutic options were discussed with the multidisciplinary team; on the basis of the single site of evident recurrent disease, the patient received 27 Gy hypofractionated adjuvant stereotactic radiotherapy (Video 1). Six months after the radiotherapy, the patient is alive and the CT scan is currently negative. The patient gave her consent to use her data.
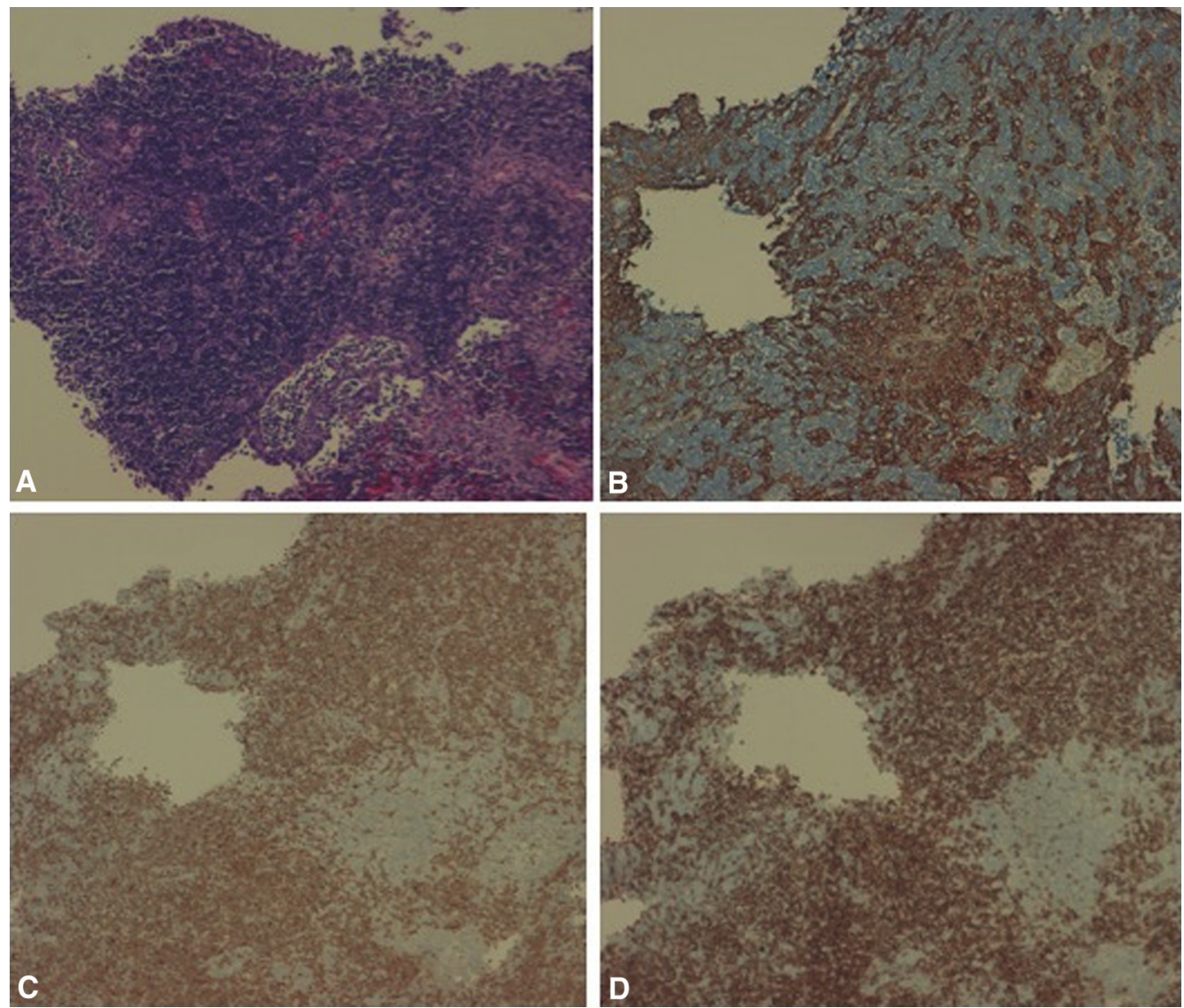

FIGURE 2. Pathology view of the metastatic node with different stains. A, Hematoxylin-eosin. B, Citokeratin pan. C, Terminal deoxynucleotidyl transferase. D, Cluster of differentiation 1a. 


\section{Single lymph-node metastasis after 10 years from radical resection of a thymoma.}

\author{
Pietro Bertoglio, Marco Lucchi, Vittorio Aprile, Greta Alì, Alfredo Muss \\ Thoracic Surgery, Department of Surgical, Medical and Molecular Pathology and Critical \\ Area, University of Pisa, Pisa, Italy \\ Pathological Anathomy, Department of Surgical, Medical and Molecular Pathology and \\ Critical Area, University of Pisa, Pisa, Italy
}

VIDEO 1. Summary of case report. Video available at: http://www. jtcvsonline.org/article/S0022-5223(17)30540-8/addons.

\section{DISCUSSION}

Thymoma is a rare cancer arising from the epithelial cells of the thymus. Recurrences after radical surgery are not common, and they are more often locoregional because of a direct spreading of the tumor. Lymph node metastases in thymomas occur in approximately $2 \%$ of the series, whereas they are more common in thymic carcinoids and carcinomas. Despite their relatively low aggressiveness, thymomas might recur several years after radical surgery, and a long-lasting follow-up is mandatory. ${ }^{1}$

We report the case of a single lymph node metastasis more than 10 years after the first intervention in a patient with B1 Masaoka stage III thymoma with no lymph node involvement at the diagnosis. Spillane and colleagues ${ }^{2}$ reported a series of late thymoma recurrences after surgery in patients affected by myasthenia gravis. In their experience, the mean disease-free interval was 12 years (range, 6-15), but in all cases recurrence was on the tumor bed or parietal pleura. Lymph node recurrence is rare, and reports are anecdotal. ${ }^{3,4}$

We have never observed metachronous nodal metastasis of thymoma. Considering the previous surgery and postoperative radiation therapy, histologic diagnosis was preferred. Consequently, Carlens mediastinoscopy procedure was our choice to achieve a complete diagnosis and to resect the metastatic node. Conversely, Pusztaszeri and colleagues ${ }^{4}$ described a simple cytologic diagnosis for cervical lymph node metastasis.

Adjuvant radiotherapy was then administered to better control the resection margins. ${ }^{5}$ Radiotherapy is considered the gold standard treatment of R1 resections; conversely, the role of chemotherapy is still not clear. ${ }^{6}$ Chemotherapy usually is reserved for the treatment of nonresectable recurrences or metastatic disease.

The Masaoka-Koga staging system is primarily used for thymomas, but recently a new staging system has been proposed $^{7}$ to create a proper TNM classification for thymic malignancies. Our report might be considered additional evidence to confirm the importance of lymphatic spread of thymomas and stress the routine evaluation of $\mathrm{N}$ classification.

\section{CONCLUSIONS}

Thymoma is a slow-growing and poorly aggressive disease that can recur a long time after surgery. Although rare, lymphatic recurrence should be suspected in the case of radiologic or clinical evidence. Careful and long-term follow-up is mandatory for all patients, and multimodality treatment, possibly including surgery, always should be considered.

\section{References}

1. Huang J, Detterbeck FC, Wang Z, Loehrer PJ Sr. Standard outcome measures for thymic malignancies. J Thorac Oncol. 2011;6(7 Suppl 3):S1691-7.

2. Spillane J, Hayward M, Hirsch NP, Taylor C, Kullmann DM, Howard RS. Late recurrent thymoma in myasthenia gravis: a case series. J Neurol Neurosurg Psychiatry. 2012;83:1030-1.

3. Xu C, Feng QF, Fan CC, Zhai YR, Chen YD, Zhang HX, et al. Patterns and predictors of recurrence after radical resection of thymoma. Radiother Oncol. 2015; 115:30-4.

4. Pusztaszeri M, Dietrich PY, Duc C, Pasquier N, McKee T, Pache JC. Fine-needle aspiration biopsy for the diagnosis of metastatic type B thymoma to lymph nodes: a case report. Diagn Cytopathol. 2014;42:683-5.

5. Luo T, Zhao H, Zhou X. The clinical features, diagnosis and management of recurrent thymoma. J Cardiothorac Surg. 2016;11:140.

6. Scorsetti M, Leo F, Trama A, D'Angelillo R, Serpico D, Macerelli M, et al. Thy moma and thymic carcinomas. Crit Rev Oncol Hematol. 2016;99:332-50.

7. Detterbeck FC, Stratton K, Giroux D, Asamura H, Crowley J, Falkson C, et al. The IASLC/ITMIG Thymic Epithelial Tumors Staging Project: proposal for an evidence-based stage classification system for the forthcoming (8th) edition of the TNM classification of malignant tumors. J Thorac Oncol. 2014;9(9 Suppl 2):S65-72. 